\title{
The Woodward-Hoffmann-De Puy Rule Revisited
}

Olalla Nieto Faza, Carlos Silva López, Rosana Álvarez, Ángel R. De Lera*

Departamento de Química Orgánica. Facultade de Química. Universidade de Vigo. Lagoas-Marcosende s/n 36200 Vigo (Spain)

qolera@uvigo.es 


\begin{tabular}{|c|c|c|c|c|c|}
\hline Structure & Electronic e & $\begin{array}{l}\text { Elec. } \\
\text { +zpve }\end{array}$ & Enthalpy & TS & Free Energy \\
\hline cis,cis-1 & $-131375,92$ & $-131295,99$ & $-131290,78$ & 25,54 & $-131316,32$ \\
\hline cis,cis-2 & $-106699,52$ & $-106637,12$ & $-106632,91$ & 23,21 & $-106656,12$ \\
\hline cis,cis-3 & $-205398,92$ & $-205265,96$ & $-205258,42$ & 30,52 & $-205288,94$ \\
\hline cis,cis-4 & $-155277,76$ & $-155194,71$ & $-155189,04$ & 26,91 & $-155215,95$ \\
\hline cis,cis-5 & $-168985,73$ & $-168928,17$ & $-168923,46$ & 24,66 & $-168948,12$ \\
\hline cis,cis-6 & $-141441,47$ & $-141368,38$ & $-141363,19$ & 25,56 & $-141388,75$ \\
\hline cis,cis-7 & $-178579,05$ & $-178496,25$ & $-178490,42$ & 27,14 & $-178517,56$ \\
\hline cis,cis-8 & $-177827,85$ & $-177759,60$ & $-177754,13$ & 26,58 & $-177780,71$ \\
\hline cis,cis-9 & $-235058,81$ & $-234994,56$ & $-234988,84$ & 27,48 & $-235016,31$ \\
\hline cis-in-1 & $-131336,65$ & $-131259,12$ & $-131253,49$ & 26,76 & $-131280,24$ \\
\hline cis-in-2 & $-106657,44$ & $-106597,42$ & $-106592,72$ & 24,58 & $-106617,29$ \\
\hline cis-in-3 & $-205357,91$ & $-205227,41$ & $-205219,14$ & 33,13 & $-205252,27$ \\
\hline cis-in-4 & $-155244,66$ & $-155163,60$ & $-155157,50$ & 28,16 & $-155185,66$ \\
\hline cis-in-5 & $-168947,20$ & $-168891,89$ & $-168886,68$ & 26,11 & $-168912,79$ \\
\hline cis-in-6 & $-141423,15$ & $-141351,73$ & $-141346,46$ & 25,69 & $-141372,15$ \\
\hline cis-in-7 & $-178548,76$ & $-178467,40$ & $-178461,35$ & 27,71 & $-178489,06$ \\
\hline cis-in-8 & $-177787,61$ & $-177721,58$ & $-177715,66$ & 28,01 & $-177743,67$ \\
\hline cis-in-9 & $-235017,50$ & $-234955,94$ & $-234949,84$ & 28,55 & $-234978,39$ \\
\hline cis-out-1 & $-131326,22$ & $-131248,94$ & $-131243,35$ & 26,68 & $-131270,03$ \\
\hline cis-out-2 & $-106644,66$ & $-106585,03$ & $-106580,37$ & 24,44 & $-106604,81$ \\
\hline cis-out-3 & $-205351,62$ & $-205221,49$ & $-205213,46$ & 31,92 & $-205245,38$ \\
\hline cis-out-4 & $-155232,02$ & $-155151,35$ & $-155145,39$ & 27,67 & $-155173,06$ \\
\hline cis-out-5 & $-168933,61$ & $-168878,36$ & $-168873,39$ & 25,37 & $-168898,75$ \\
\hline cis-out-6 & $-141408,05$ & $-141336,77$ & $-141331,61$ & 25,48 & $-141357,09$ \\
\hline cis-out-7 & $-178531,27$ & $-178450,45$ & $-178444,35$ & 27,91 & $-178472,26$ \\
\hline cis-out-8 & $-177770,41$ & $-177705,17$ & $-177699,33$ & 27,69 & $-177727,02$ \\
\hline cis-out-9 & $-235000,57$ & $-234939,45$ & $-234933,41$ & 28,09 & $-234961,50$ \\
\hline trans,cis-1 & $-131377,67$ & $-131297,87$ & $-131292,61$ & 25,64 & $-131318,25$ \\
\hline
\end{tabular}




\begin{tabular}{|c|c|c|c|c|c|}
\hline Structure & Electronic & $\begin{array}{l}\text { Elec. } \\
\text { y +zpve }\end{array}$ & Enthalpy & TS & Free Energy \\
\hline trans,cis-2 & $-106700,41$ & $-106638,10$ & $-106633,86$ & 23,27 & $-106657,13$ \\
\hline trans,cis-3 & $-205403,48$ & $-205270,85$ & $-205263,13$ & 31,41 & $-205294,55$ \\
\hline trans,cis-4 & $-155277,29$ & $-155194,41$ & $-155188,66$ & 27,10 & $-155215,77$ \\
\hline trans,cis-5 & $-168988,77$ & $-168931,37$ & $-168926,59$ & 24,79 & $-168951,38$ \\
\hline trans,cis-6 & $-141444,57$ & $-141371,44$ & $-141366,33$ & 25,25 & $-141391,58$ \\
\hline trans,cis-7 & $-178581,97$ & $-178499,30$ & $-178493,40$ & 27,41 & $-178520,81$ \\
\hline trans,cis-8 & $-177829,87$ & $-177761,79$ & $-177756,26$ & 26,71 & $-177782,97$ \\
\hline trans,cis-9 & $-235064,44$ & $-235000,29$ & $-234994,53$ & 27,49 & $-235022,02$ \\
\hline trans-in-1 & $-131313,60$ & $-131236,29$ & $-131230,61$ & 27,02 & $-131257,63$ \\
\hline trans-in-2 & $-106639,15$ & $-106579,58$ & $-106574,86$ & 24,70 & $-106599,55$ \\
\hline trans-in-3 & $-205335,67$ & $-205205,69$ & $-205197,46$ & 32,75 & $-205230,22$ \\
\hline trans-in-4 & $-155218,92$ & $-155138,54$ & $-155132,40$ & 28,27 & $-155160,67$ \\
\hline trans-in-5 & $-168921,72$ & $-168866,95$ & $-168861,72$ & 26,13 & $-168887,85$ \\
\hline trans-in-6 & $-141392,34$ & $-141321,53$ & $-141316,21$ & 25,87 & $-141342,08$ \\
\hline trans-in-7 & $-178523,70$ & $-178443,34$ & $-178437,10$ & 28,31 & $-178465,41$ \\
\hline \multicolumn{6}{|l|}{ trans-in-8 } \\
\hline trans-in-9 & $-235000,16$ & $-234939,00$ & $-234933,26$ & 27,18 & $-234960,44$ \\
\hline trans-out-1 & $-131343,67$ & $-131266,21$ & $-131260,43$ & 27,56 & $-131287,99$ \\
\hline trans-out-2 & $-106660,15$ & $-106600,22$ & $-106595,46$ & 24,83 & $-106620,30$ \\
\hline trans-out-3 & $-205373,41$ & $-205242,85$ & $-205234,67$ & 33,02 & $-205267,69$ \\
\hline trans-out-4 & $-155251,84$ & $-155170,72$ & $-155164,68$ & 28,10 & $-155192,79$ \\
\hline trans-out-5 & $-168952,61$ & $-168897,17$ & $-168891,96$ & 26,40 & $-168918,36$ \\
\hline trans-out-6 & $-141425,18$ & $-141353,59$ & $-141348,23$ & 26,09 & $-141374,32$ \\
\hline trans-out-7 & $-178556,58$ & $-178475,42$ & $-178469,16$ & 28,74 & $-178497,90$ \\
\hline trans-out-8 & $-177796,13$ & $-177730,01$ & $-177724,19$ & 27,69 & $-177751,88$ \\
\hline trans-out-9 & $-235025,94$ & $-234964,19$ & $-234958,05$ & 28,77 & $-234986,82$ \\
\hline
\end{tabular}

Table 1. Thermodynamic information for the structures in Figure 2 (gas phase), computed using B3LYP with the SKBJ electronic core potential with two $d$ and one $f$ functions added for the $\mathrm{Br}$, and the 6-311++G** basis set for the other atoms. All the data are in $\mathrm{kcal} / \mathrm{mol}$. 


\begin{tabular}{|c|c|c|c|c|c|}
\hline Structure & Electronic & $\begin{array}{l}\text { Elec. } \\
\text { +zpve }\end{array}$ & Enthalpy & TS & Free Energy \\
\hline cis,cis-1 & $-131376,31$ & $-131296,38$ & $-131291,16$ & 25,55 & $-131316,71$ \\
\hline cis,cis-2 & $-106700,07$ & $-106637,67$ & $-106633,45$ & 23,22 & $-106656,67$ \\
\hline cis,cis-3 & $-205399,19$ & $-205266,23$ & $-205258,69$ & 30,53 & $-205289,22$ \\
\hline cis,cis-4 & $-155278,08$ & $-155195,03$ & $-155189,35$ & 26,91 & $-155216,27$ \\
\hline cis,cis-5 & $-168986,62$ & $-168929,06$ & $-168924,34$ & 24,66 & $-168949,00$ \\
\hline cis,cis-6 & $-141441,61$ & $-141368,56$ & $-141363,34$ & 25,66 & $-141389,01$ \\
\hline cis,cis-7 & $-178578,93$ & $-178496,16$ & $-178490,32$ & 27,20 & $-178517,52$ \\
\hline cis,cis-8 & $-177830,04$ & $-177761,79$ & $-177756,32$ & 26,53 & $-177782,85$ \\
\hline cis,cis-9 & $-235060,78$ & $-234996,55$ & $-234990,82$ & 27,50 & $-235018,32$ \\
\hline cis-in-1 & $-131343,68$ & $-131265,87$ & $-131260,21$ & 27,03 & $-131287,24$ \\
\hline cis-in-2 & $-106664,00$ & $-106603,78$ & $-106599,06$ & 24,76 & $-106623,83$ \\
\hline cis-in-3 & $-205362,76$ & $-205232,08$ & $-205223,82$ & 33,14 & $-205256,96$ \\
\hline cis-in-4 & $-155248,83$ & $-155167,61$ & $-155161,55$ & 28,08 & $-155189,63$ \\
\hline cis-in-5 & $-168952,56$ & $-168897,04$ & $-168891,87$ & 26,04 & $-168917,91$ \\
\hline cis-in-6 & $-141426,09$ & $-141354,71$ & $-141349,35$ & 26,01 & $-141375,36$ \\
\hline cis-in-7 & $-178551,81$ & $-178470,36$ & $-178464,35$ & 27,58 & $-178491,93$ \\
\hline cis-in-8 & $-177790,90$ & $-177724,88$ & $-177718,95$ & 27,99 & $-177746,94$ \\
\hline cis-in-9 & $-235021,40$ & $-234959,86$ & $-234953,74$ & 28,53 & $-234982,27$ \\
\hline trans,cis-1 & $-131378,44$ & $-131298,65$ & $-131293,38$ & 25,67 & $-131319,05$ \\
\hline trans,cis-2 & $-106700,95$ & $-106638,66$ & $-106634,40$ & 23,28 & $-106657,69$ \\
\hline trans,cis-3 & $-205403,95$ & $-205271,33$ & $-205263,61$ & 31,41 & $-205295,02$ \\
\hline trans,cis-4 & $-155277,78$ & $-155194,92$ & $-155189,16$ & 27,11 & $-155216,28$ \\
\hline trans,cis-5 & $-168989,14$ & $-168931,76$ & $-168926,97$ & 24,81 & $-168951,79$ \\
\hline trans,cis-6 & $-141445,08$ & $-141371,98$ & $-141366,86$ & 25,28 & $-141392,14$ \\
\hline trans,cis-7 & $-178582,16$ & $-178499,52$ & $-178493,61$ & 27,44 & $-178521,05$ \\
\hline trans,cis-8 & $-177830,95$ & $-177762,85$ & $-177757,33$ & 26,67 & $-177784,01$ \\
\hline trans,cis-9 & $-235065,53$ & $-235001,40$ & $-234995,64$ & 27,48 & $-235023,12$ \\
\hline trans-in-1 & $-131325,59$ & $-131248,33$ & $-131242,62$ & 27,37 & $-131269,99$ \\
\hline trans-in-2 & $-106650,28$ & $-106590,63$ & $-106585,94$ & 24,75 & $-106610,69$ \\
\hline
\end{tabular}




\begin{tabular}{|c|c|c|c|c|c|}
\hline Structure & Electronic energy & $\begin{array}{l}\text { Elec. } \\
\text { +zpve }\end{array}$ & Enthalpy & TS & Free Energy \\
\hline trans-in-3 & $-205345,36$ & $-205215,46$ & $-205207,20$ & 33,21 & $-205240,41$ \\
\hline trans-in-4 & $-155225,84$ & $-155145,39$ & $-155139,26$ & 28,32 & $-155167,58$ \\
\hline trans-in-5 & $-168925,62$ & $-168870,76$ & $-168865,58$ & 26,02 & $-168891,60$ \\
\hline trans-in-6 & $-141397,72$ & $-141326,96$ & $-141321,64$ & 25,82 & $-141347,47$ \\
\hline trans-in-7 & $-178530,32$ & $-178450,06$ & $-178443,80$ & 28,43 & $-178472,23$ \\
\hline trans-in-8 & $-177768,13$ & $-177703,01$ & $-177696,99$ & 28,31 & $-177725,30$ \\
\hline trans-in-9 & $-235030,61$ & $-234968,94$ & $-234962,83$ & 28,60 & $-234991,43$ \\
\hline trans-out-1 & $-131353,73$ & $-131275,77$ & $-131270,12$ & 27,15 & $-131297,27$ \\
\hline trans-out-2 & $-106666,07$ & $-106605,98$ & $-106601,20$ & 25,04 & $-106626,25$ \\
\hline trans-out-3 & $-205379,59$ & $-205248,75$ & $-205240,63$ & 32,92 & $-205273,55$ \\
\hline trans-out-4 & $-155258,58$ & $-155177,16$ & $-155171,17$ & 28,07 & $-155199,24$ \\
\hline trans-out-5 & $-168960,50$ & $-168904,69$ & $-168899,61$ & 26,02 & $-168925,63$ \\
\hline trans-out-6 & $-141431,54$ & $-141359,73$ & $-141354,44$ & 26,00 & $-141380,44$ \\
\hline trans-out-7 & $-178563,81$ & $-178482,50$ & $-178476,30$ & 28,56 & $-178504,86$ \\
\hline trans-out-8 & $-177800,62$ & $-177734,51$ & $-177728,68$ & 27,74 & $-177756,42$ \\
\hline trans-out-9 & $-235030,03$ & $-234968,35$ & $-234962,24$ & 28,64 & $-234990,87$ \\
\hline
\end{tabular}

Table 2. Table 1. Thermodynamic information for the structures in Figure 2 (SCRF with MeOH, Onsager method), computed using B3LYP with the SKBJ electronic core potential with two d and one $f$ functions added for the $\mathrm{Br}$, and the $6-311++\mathrm{G}^{* *}$ basis set for the other atoms. All the data are in $\mathrm{kcal} / \mathrm{mol}$. 


\begin{tabular}{|c|c|c|c|c|c|}
\hline Structure & $\mathbf{r} 23$ & r16 & a213 & d4213 & d5312 \\
\hline cis,cis-1 & 1,524 & 1,942 & 60,7 & 111,3 & 111,3 \\
\hline cis,cis-2 & 1,518 & 1,94 & 60,7 & 109 & 106,3 \\
\hline cis,cis-3 & 1,527 & 1,945 & 60,7 & 116 & 119,6 \\
\hline cis,cis-4 & 1,536 & 1,939 & 61,3 & 110,9 & 110 \\
\hline cis,cis-5 & 1,505 & 1,926 & 59,8 & 109,1 & 107,4 \\
\hline cis,cis-6 & 1,513 & 1,935 & 59,7 & 111,3 & 108,7 \\
\hline cis,cis-7 & 1,507 & 1,932 & 59,6 & 109,6 & 105,3 \\
\hline cis,cis-8 & 1,544 & 1,939 & 61,8 & 111,5 & 108,5 \\
\hline cis,cis-9 & 1,527 & 1,92 & 60,8 & 113,9 & 111,2 \\
\hline cis-in-1 & 2,317 & 2,465 & 110,9 & 52,8 & 52,8 \\
\hline cis-in-2 & 2,256 & 2,468 & 107,3 & 54,8 & 40,7 \\
\hline cis-in-3 & 2,348 & 2,479 & 113 & 55,5 & 68,5 \\
\hline \multicolumn{6}{|l|}{ cis-in-4 } \\
\hline cis-in-5 & 2,29 & 2,403 & 109,3 & 54,6 & 49,9 \\
\hline cis-in-6 & 2,096 & 2,215 & 94 & 71,7 & 84,2 \\
\hline cis-in-7 & 2,201 & 2,343 & 101,6 & 58,7 & 70,7 \\
\hline cis-in-8 & 2,354 & 2,343 & 112,1 & 53,7 & 56,8 \\
\hline cis-in-9 & 2,424 & 2,243 & 116 & 59,5 & 54,2 \\
\hline cis-out-1 & 1,932 & 2,648 & 85,9 & 128,2 & 128,2 \\
\hline cis-out-2 & 1,932 & 2,752 & 86,7 & 125,3 & 124,9 \\
\hline cis-out-3 & 1,909 & 2,656 & 84,4 & 126,8 & 131,8 \\
\hline cis-out-4 & 2,073 & 2,419 & 92,5 & 133,3 & 130,9 \\
\hline cis-out-5 & 2,027 & 2,411 & 90,7 & 138 & 137,7 \\
\hline cis-out-6 & 1,969 & 2,281 & 85,9 & 136,8 & 130,6 \\
\hline cis-out-7 & 1,932 & 2,136 & 86,7 & 125,3 & 165,2 \\
\hline cis-out-8 & 2,192 & 2,269 & 98,3 & 139 & 154,8 \\
\hline cis-out-9 & 2,157 & 2,218 & 96,1 & 141,4 & 154,9 \\
\hline trans,cis-1 & 1,527 & 1,942 & 61,2 & 113,6 & 113,6 \\
\hline trans,cis-2 & 1,518 & 1,939 & 60,8 & 111 & 109,1 \\
\hline
\end{tabular}




\begin{tabular}{|c|c|c|c|c|c|}
\hline trans,cis-3 & 1,534 & 1,946 & 61,6 & 117,9 & 120 \\
\hline Structure & r23 & r16 & a213 & d4213 & d5312 \\
\hline trans,cis-4 & 1,529 & 1,94 & 61,3 & 114,6 & 115,4 \\
\hline trans,cis-5 & 1,506 & 1,933 & 60,2 & 111,5 & 109,2 \\
\hline trans,cis-6 & 1,526 & 1,938 & 61,1 & 112,5 & 109,6 \\
\hline trans,cis-7 & 1,509 & 1,938 & 60,1 & 111,9 & 107,2 \\
\hline trans,cis-8 & 1,544 & 1,931 & 62 & 113,3 & 111,5 \\
\hline trans,cis-9 & 1,533 & 1,928 & 61,7 & 113,7 & 110,2 \\
\hline trans-in-1 & 1,9 & 2,907 & 84,8 & 77,9 & 77,9 \\
\hline trans-in-2 & 1,933 & 2,866 & 87,1 & 74,1 & 56 \\
\hline trans-in-3 & 1,9 & 2,97 & 84,8 & 79,2 & 92,4 \\
\hline trans-in-4 & 2,109 & 2,599 & 95,5 & 64,7 & 66,1 \\
\hline trans-in-5 & 2,022 & 2,667 & 91,2 & 62,3 & 74 \\
\hline trans-in-6 & 2,181 & 2,323 & 98 & 53,3 & 79,2 \\
\hline trans-in-7 & 2,095 & 2,481 & 94,3 & 60,2 & 79,5 \\
\hline \multicolumn{6}{|l|}{ trans-in-8 } \\
\hline trans-in-9 & 2,434 & 2,038 & 110,3 & 11,7 & 79,8 \\
\hline trans-out-1 & 2,216 & 2,48 & 104,7 & 139,2 & 139,2 \\
\hline trans-out-2 & 2,21 & 2,454 & 104,4 & 134,6 & 137 \\
\hline trans-out-3 & 2,193 & 2,483 & 103,1 & 138,3 & 139,1 \\
\hline trans-out-4 & 2,228 & 2,403 & 104,4 & 137,1 & 132,9 \\
\hline trans-out-5 & 2,205 & 2,464 & 104,3 & 142,8 & 140,2 \\
\hline trans-out-6 & 2,038 & 2,257 & 91,6 & 130,7 & 126,7 \\
\hline trans-out-7 & 2,135 & 2,374 & 98,6 & 137,3 & 131,6 \\
\hline trans-out-8 & 2,253 & 2,328 & 105,8 & 131 & 133,4 \\
\hline trans-out-9 & 2,233 & 2,294 & 104,6 & 131,2 & 135,8 \\
\hline
\end{tabular}

Table 3. The most relevant geometric parameters for the structures in Figure 2 (gas phase). The bond distances are in $\AA$, and the angles and dihedrals in degrees. 


\begin{tabular}{|c|c|c|c|c|c|}
\hline Structure & r23 & r16 & a213 & d4213 & d5312 \\
\hline cis,cis-1 & 1,524 & 1,945 & 60,7 & 111,3 & 111,3 \\
\hline cis,cis-2 & 1,518 & 1,945 & 60,7 & 108,9 & 106,2 \\
\hline cis,cis-3 & 1,526 & 1,947 & 60,7 & 115,9 & 119,6 \\
\hline cis,cis-4 & 1,536 & 1,941 & 61,3 & 110,9 & 110,0 \\
\hline cis,cis-5 & 1,505 & 1,929 & 59,8 & 109,3 & 107,4 \\
\hline cis,cis-6 & 1,512 & 1,937 & 59,7 & 111,3 & 108,7 \\
\hline cis,cis-7 & 1,513 & 2,999 & 28,9 & 0,7 & 59,3 \\
\hline cis,cis-8 & 1,548 & 1,940 & 61,9 & 112,1 & 108,9 \\
\hline cis,cis-9 & 1,529 & 1,921 & 60,9 & 114,2 & 111,6 \\
\hline cis-in-1 & 2,186 & 2,519 & 102,2 & 60,9 & 60,9 \\
\hline cis-in-2 & 2,180 & 2,585 & 102,7 & 59,3 & 42,9 \\
\hline cis-in-3 & 2,258 & 2,521 & 106,7 & 62,4 & 71,3 \\
\hline cis-in-4 & 2,251 & 2,435 & 105,4 & 58,9 & 60,8 \\
\hline cis-in-5 & 2,194 & 2,426 & 102,7 & 59,5 & 58,0 \\
\hline cis-in-6 & 2,001 & 2,187 & 88,3 & 77,3 & 88,8 \\
\hline cis-in-7 & 2,079 & 2,285 & 93,6 & 67,3 & 75,1 \\
\hline cis-in-8 & 2,366 & 2,372 & 113,1 & 57,9 & 53,7 \\
\hline cis-in-9 & 2,436 & 2,238 & 116,7 & 60,2 & 56,5 \\
\hline trans,cis-1 & 1,703 & 3,036 & 73,9 & 91,9 & 91,9 \\
\hline trans,cis-2 & 1,732 & 2,950 & 75,8 & 85,9 & 75,5 \\
\hline trans,cis-3 & 1,750 & 3,109 & 76,7 & 91,2 & 98,9 \\
\hline trans,cis-4 & 1,809 & 2,839 & 79,2 & 86,4 & 84,5 \\
\hline trans,cis-5 & 1,884 & 2,758 & 83,4 & 73,5 & 80,7 \\
\hline trans,cis-6 & 2,135 & 2,297 & 95,0 & 61,3 & 80,6 \\
\hline trans,cis-7 & 1,929 & 2,564 & 85,0 & 75,0 & 87,0 \\
\hline trans,cis-8 & 1,947 & 2,916 & 87,4 & 82,2 & 72,9 \\
\hline trans,cis-9 & 1,466 & 3,182 & 15,0 & 84,0 & 169,2 \\
\hline trans-in-1 & 1,527 & 1,948 & 61,3 & 113,6 & 113,6 \\
\hline
\end{tabular}




\begin{tabular}{lccccc} 
trans-in-2 & 1,518 & 1,943 & 60,9 & 111,1 & 109,1 \\
trans-in-3 & 1,534 & 1,951 & 61,6 & 117,9 & 120,0 \\
Structure & $\mathbf{r 2 3}$ & $\mathbf{r 1 6}$ & $\mathbf{a 2 1 3}$ & $\mathbf{d 4 2 1 3}$ & $\mathbf{d 5 3 1 2}$ \\
trans-in-4 & 1,530 & 1,945 & 61,3 & 114,5 & 115,3 \\
trans-in-5 & 1,506 & 1,935 & 60,2 & 111,8 & 109,6 \\
trans-in-6 & 1,526 & 1,941 & 61,1 & 112,5 & 109,4 \\
trans-in-7 & 1,509 & 1,941 & 60,1 & 111,9 & 107,1 \\
trans-in-8 & 1,545 & 1,931 & 62,0 & 113,6 & 112,3 \\
trans-in-9 & 1,535 & 1,927 & 61,8 & 113,9 & 110,8 \\
trans-out-1 & 2,035 & 2,555 & 93,2 & 124,6 & 124,6 \\
trans-out-2 & 2,161 & 2,575 & 101,6 & 130,8 & 133,5 \\
trans-out-3 & 2,097 & 2,544 & 97,1 & 129,9 & 129,8 \\
\hline trans-out-4 & 2,081 & 2,438 & 95,2 & 125,2 & 122,2 \\
trans-out-5 & 2,061 & 2,485 & 94,9 & 129,7 & 130,6 \\
trans-out-6 & 1,903 & 2,259 & 83,8 & 123,1 & 122,6 \\
trans-out-7 & 1,988 & 2,349 & 89,4 & 126,9 & 123,6 \\
trans-out-8 & 1,410 & 5,340 & 26,5 & 48,9 & 95,6 \\
trans-out-9 & 2,239 & 2,272 & 104,5 & 130,9 & 139,5 \\
& & & & & \\
\hline
\end{tabular}

Table 4. The most relevant geometric parameters for the structures in Figure 2 (SCRF with MeOH, Onsager method). The bond distances are in $\AA$, and the angles and dihedrals in degrees.

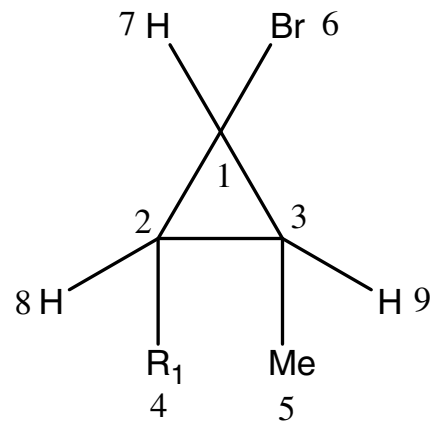

Figure 1. Numbering used in Table 3 and Table 4. 